Pacific Journal of Mathematics

ON SOLVABLE $\boldsymbol{O}^{*}$-GROUP 


\title{
ON SOLVABLE O*-GROUPS
}

\author{
D. P. MinASSIAN
}

The purpose of this paper is to prove the existence of $0^{*}$-groups of arbitrary solvable length, as well as of nonsolvable $0 *$-groups.

By a partial order for a group $G$ we mean a reflexive, antisymmetric and transitive relation, $\leqq$, on $G$ such that if $g$ and $h$ are elements of $G$ and $g \leqq h$, then $x g y \leqq x h y$ for all $x$ and $y$ in $G$. If also any two elements $g$ and $h$ of $G$ are comparable (i.e., either $g \leqq h$ or $h \leqq g$ ), then the partial order for $G$ is called a total (or full, or linear) order. The group $G$ is an $O^{*}$-group if any partial order for $G$ is included in some total order for $G$.

A group $G$ is solvable of length $n$, where $n$ is a positive integer, if the derived chain of $G$ reaches the unit subgroup, $E$, in $n$ steps:

$$
G=G^{1} \supsetneq G^{2} \supsetneq \cdots \supsetneq G^{n} \supsetneq G^{n+1}=E,
$$

where $G^{i+1}$ is the derived group of $G^{i}$ (denoted below by $G^{i+1}=\left[G^{i}, G^{i}\right]$ ).

It has been shown that non-abelian free groups are not $O^{*}$-groups ([1], [2], [3], [4], [6]). Further, Kargapolov [5] and Kargapolov, Kokorin and Kopytov [6] have produced solvable groups which are not $\mathrm{O}^{*}$-groups even though they admit a full order: these are the free $r$-step solvable groups on $k$ generators for $r \geqq 3$ and $k \geqq 2$. In view of these results one may ask if there exist solvable $0^{*}$-groups of arbitrary length, and nonsolvable $\mathrm{O}^{*}$-groups. The answers are affirmative.

THEOREM. For every positive integer $m$ there exists an $O^{*}$-group $G$ that is solvable of length $m$.

Proof. Let $F$ be the free group on $k$ generators for some fixed $k \geqq 2$. Let $F_{i}$ be the $i$ th term in the lower central series for $F$, where $F_{1}=F$, and let $F^{i}$ be the $i$ th derived group for $F$, where $F^{1}$ $=F$. Consider $F / F_{i}$, the free nilpotent group of class $i$ with $k$ generators. By varying $i$ we shall obtain the desired groups $G$ of the theorem.

We first claim that $F / F_{i}$ is torsion-free for every positive integer $i$. If not, then for some $i$ there exists an element $a \in F$ and a positive integer $p$ such that $a \notin F_{i}$, but $a^{p} \in F_{i}$. Now $a \in F_{h}-F_{h+1}$ for some positive integer $h \leqq i-1$. Thus $a^{p} \in F_{i} \subseteq F_{h+1}$, and so $F_{h} / F_{h+1}$ is not torsion-free. On the other hand, Witt's theorem (see, e.g., [8, p. 41]) states that $F_{h} / F_{h+1}$ is a free abelian group (and hence torsion- 
free), a contradiction. Thus $F / F_{i}$ is torsion-free, as claimed.

Malcev [9] has shown that a torsion-free nilpotent group is an $\mathrm{O}^{*}$-group. Hence $F / F_{i}$ is an $\mathrm{O}^{*}$-group for every positive integer $i$. Now for every such $i$ the solvable length of $F / F_{i}$ is finite, since $F / F_{i}$ is nilpotent. Thus we shall complete our proof by establishing the following lemma.

LEMma. For every positive integer $m$, there exists an integer $n$ such that solvable length of $F / F_{n}$ is $m$.

Proof. We first note that for every positive integer $i$, there exists an integer $j$ such that $F_{j} \nsupseteq F^{i}$. This follows from the fact that $F^{i} \neq E$ for each $i$ (hence $F$ is not solvable), together with the theorem of Magnus (cf. [8, p. 38]) which asserts that $\bigcap_{i=1}^{\infty} F_{i}=E$. We next show that for each $i$ and $j$,

$$
\left(F / F_{j}\right)^{i}=F^{i} F_{j} / F_{j} .
$$

Indeed, it is readily seen that if $A$ and $B$ are subgroups of a group $G$ and if $B$ is invariant under conjugation by elements of $A$, then $(A B / B)^{2}=A^{2} B / B$. From this, an induction on $i$ shows that for a normal subgroup $N$ of a group $G$ it is true that $(G / N)^{i}=G^{i} N / N$ for all $i$, which implies the desired result.

Note that for each $i$ there exists $J$ such that for $j \geqq J$, the solvable length of $F / F_{j}$ exceeds $i$. This follows from (1) and the fact that, by our first assertion, we can choose $J$ such that $F_{J} \nsupseteq F^{i}$. In particular, then, the solvable length of $F / F_{j}$ is unbounded with increasing $j$. Note also that the solvable length of $F / F_{j+1}$ exceeds the solvable length of $F / F_{j}$ by at most 1 . For if $F / F_{j}$ is solvable of length $r-1$, then $\left(F / F_{j}\right)^{r}=E$. Thus, by (1) we have $F^{r} F_{j} / F_{j}=E$, which implies $F^{r} \subseteq F_{j}$. On the other hand, $F / F_{j+1}$ has solvable length $\leqq r$ since (again using (1))

$$
\begin{aligned}
\left(F / F_{j+1}\right)^{r+1} & =\left[\left(F / F_{j+1}\right)^{r},\left(F / F_{j+1}\right)^{r}\right] \\
& =\left[F^{r} F_{j+1} / F_{j+1}, F^{r} F_{j+1} / F_{j+1}\right] \subseteq\left[F_{j} / F_{j+1}, F_{j} / F_{j+1}\right]=E,
\end{aligned}
$$

where $\subseteq$ holds since both $F^{r}$ and $F_{j+1}$ are subsets of $F_{j}$, and the final equality derives from the fact that $F_{j} / F_{j+1}$ is abelian by Witt's theorem (above). The lemma follows at once from these results and the fact that $F / F_{2}=F_{1} / F_{2}$ has solvable length 1 by Witt's theorem.

The proof of the theorem is now complete.

CoRollary. There exist nonsolvable $O^{*}$-groups.

Proof. Kargapolov [5] and Kokorin [7] have shown that the re- 
stricted direct product of $\mathrm{O}^{*}$-groups is an $\mathrm{O}^{*}$-group. Thus the restricted direct product, $G=\prod_{i=1}^{\infty} F / F_{i}$, of the groups $F / F_{i}$ is an $\mathrm{O}^{*}$-group. If $G$ were solvable of length $m$, then each $F / F_{i}$ would have solvable length $\leqq m$; for if a subgroup $H \subseteq G$, then $H^{k} \subseteq G^{k}$ for every $k$. Since this contradicts the fact noted above that the solvable length of $F / F_{j}$ is unbounded with increasing $j, G$ is a non-solvable $\mathrm{O}^{*}$-group.

Note. The mapping $\varphi$ of $F$ into the unrestricted (or complete) direct product, $\prod_{i=1}^{\infty} F / F_{i}$, of the groups $F / F_{i}$ given by

$$
\varphi(a)=\left(a F_{1}, \cdots, a F_{n}, \cdots\right) \text { for every } a \in F
$$

is a monomorphism by Magnus' theorem, above. Since $F$ is not an $0^{*}$-group (see [1], [4], or [6]), we have an immediate example of a subdirect product of $\mathrm{O}^{*}$-groups which is not itself an $\mathrm{O}^{*}$-group. (In [5], Kargapolov uses some of the groups $F / F_{i}$ to show that the class of $\mathrm{O}^{*}$-groups is not closed under formation of unrestricted direct products.)

Acknowledgement. I acknowledge the generously donated help of Professor E. F. Krause (University of Michigan), my adviser for the doctoral dissertation on which this paper is based.

\section{REFERENCES}

1. L. Fuchs, On orderable groups, Proc. Internat. Conf. Theory of Groups (Canberra, 1965), 89-98, Gordon and Breach, New York, 1967.

2. L. Fuchs and E. Sasiada, Note on orderable groups, Annales Universitatis Scientiarum Budapestinensis de Rolando Eotvos Nominatae, Sectio Mathematica (1964), 13-17.

3. W. C. Holland, Extensions of Ordered Algebraic Structures, Doctoral Thesis, Tulane Univerity, 1961.

4. H. Hollister, Groups in which every maximal partial order is isolated, Proc. Amer. Math. Soc., 19 (1968), 467-469.

5. M. I. Kargapolov, Fully orderable groups (Russian), Algebra i Logika Sem., (6) 2 (1963), 5-14.

6. M. I. Kargapolov, A. I. Kokorin, and V. M. Kopytov, On the theory of orderable groups (Russian), Algebra i Logika Sem., (6) 4 (1965), 21-27.

7. A. I. Kokorin, Ordering a direct product of ordered groups (Russian), Ural. Gos. Univ. Mat. Zap., (3) 4 (1963), 95-96.

8. A. G. Kurosh. The Theory of Groups, vol. ii, Chelsea, New York, 1956.

9. A. I. Malcev, On the completion of group order (Russian), Trudy Mat. Inst. Steklov, 38 (1951), 173-175.

Received August 25, 1970 and in revised form April 12, 1971.

Butler UNIVERSity 



\section{PACIFIC JOURNAL OF MATHEMATICS}

\section{EDITORS}

H. SAMELSON

Stanford University

Stanford, California 94305

C. R. HobBY

University of Washington

Seattle, Washington 98105
J. DugundjI

Department of Mathematics

University of Southern California

Los Angeles, California 90007

RICHARD ARENS

University of California

Los Angeles, California 90024

\section{ASSOCIATE EDITORS}

E. F. BECKENBACH

B. H. NeUMaNN

F. WOLF

K. YoshidA

\section{SUPPORTING INSTITUTIONS}

UNIVERSITY OF BRITISH COLUMBIA

CALIFORNIA INSTITUTE OF TECHNOLOGY

UNIVERSITY OF CALIFORNIA

MONTANA STATE UNIVERSITY

UNIVERSITY OF NEVADA

NEW MEXICO STATE UNIVERSITY

OREGON STATE UNIVERSITY

UNIVERSITY OF OREGON

OSAKA UNIVERSITY
UNIVERSITY OF SOUTHERN CALIFORNIA STANFORD UNIVERSITY

UNIVERSITY OF TOKYO

UNIVERSITY OF UTAH

WASHINGTON STATE UNIVERSITY UNIVERSITY OF WASHINGTON

$\stackrel{*}{*} \stackrel{*}{*} \stackrel{*}{*}$ AMERICAN MATHEMATICAL SOCIETY
NAVAL WEAPONS CENTER 


\section{Pacific Journal of Mathematics}

Vol. 39 , No. 1

May, 1971

Charles A. Akemann, A Gelfand representation theory for $C^{*}$-algebras ....

Sorrell Berman, Spectral theory for a first-order symmetric system of

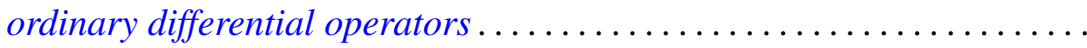

Robert L. Bernhardt, III, On splitting in hereditary torsion theories ........

J. L. Brenner, Geršgorin theorems, regularity theorems, and bounds for determinants of partitioned matrices. II. Some determinantal identities ..........................................

Robert Morgan Brooks, On representing $F^{*}$-algebras .............. 51

Lawrence Gerald Brown, Extensions of topological groups........... 71

Arnold Barry Calica, Reversible homeomorphisms of the real line ........ 79

J. T. Chambers and Shinnosuke Oharu, Semi-groups of local Lipschitzians in

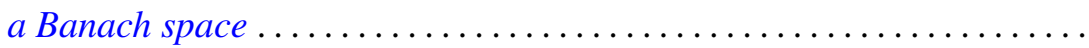

Thomas J. Cheatham, Finite dimensional torsion free rings .............

Byron C. Drachman and David Paul Kraines, A duality between

transpotence elements and Massey products ...................

Richard D. Duncan, Integral representation of excessive functions of a

Markov process ......................................

George A. Elliott, An extension of some results of Takesaki in the reduction

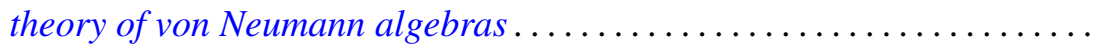

Peter C. Fishburn and Joel Spencer, Directed graphs as unions of partial

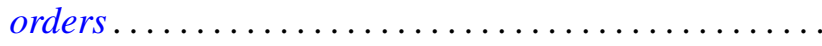

Howard Edwin Gorman, Zero divisors in differential rings ...

Maurice Heins, A note on the Löwner differential equations...

Louis Melvin Herman, Semi-orthogonality in Rickart rings. .

David Jacobson and Kenneth S. Williams, On the solution of linear G.C.D.

equations

Michael Joseph Kallaher, On rank 3 projective planes ... . .

Donald Paul Minassian, On solvable $O^{*}$-groups ...........

Nils Øvrelid, Generators of the maximal ideals of $A(\bar{D})$

Mohan S. Putcha and Julian Weissglass, A semilattice decomposition into

semigroups having at most one idempotent ............

Robert Raphael, Rings of quotients and $\pi$-regularity ....

J. A. Siddiqi, Infinite matrices summing every almost periodic sequence. .

Raymond Earl Smithson, Uniform convergence for multifunctions ...

Thomas Paul Whaley, Mulitplicity type and congruence relations in

universal algebras...

Roger Allen Wiegand, Globalization theorems for locally finitely generated modules... 